\title{
Design of Computer Application Building Automation Control System
}

\author{
Kai Cheng, ${ }^{1, *}$, Shuai Ren ${ }^{1}$, and Tariq Abu Zalata ${ }^{2}$ \\ ${ }^{I}$ Department of Information Technology, Henan Institute of Education, Zhengzhou 450046, China \\ ${ }^{2}$ Electrical Engineering Department, College of Engineering, University of Anbar, Baghdad, Iraq
}

\begin{abstract}
The object of this research is building automation control system and to design the system by means of the computer application. First, I analyze the operating principle of the automatic monitored control system based on the computer technology, and I find that with the help of Ethernet switching engineering we can solve the problem about high network load and transmission delay of traditional building automation control system. At the same time, I design the overall structure of the automatic monitored control system. Then I learn the hardware control system and the implementation of the software, and the mainly representative object is the central air-conditioning control system. As the core component of the control system, PLC's hardware and transducer are chosen. And I design the process of software control, data acquisition, communication device and other modules. Meanwhile, I finish the design of the procedure of PLC master control module and realize the software monitoring and control with the host computer software. Finally, I explore the remote monitoring software based on internet, and improve some problem like the spread of information during the monitoring in the past and develop the auxiliary function of short message reminder with the end of the integrated structure, idea and the structure based on the computer application and the design of the key part.
\end{abstract}

Keywords: Building automation control system, PLC, host computer, remote monitoring software.

\section{INTRODUCTION}

With the rapid development and wide application of the computer, the automatic technology also makes a great progress, including the emerging of building automation control system. The building automation control system integrates the electronic systems in a building into a computer platform which offers a comprehensive service of multifunction like monitor and alarm. And it also accomplishes the information sharing and communications among subsystems and meanwhile manages subsystems reasonably to realize the automated monitoring and control. The goal is to improve work efficiency and provide comfortable environment; to reduce the resource costs as much as possible in a reasonable way; to adapt to various environment and prevent and cope with the emergency. According to the current situation of incompatible communication protocol and unstable data transmission in domestic building system, I start the development of automatic monitored control system based on the computer technology.

\section{THE OVERALL STRUCTURE DESIGN OF BUILD- ING AUTOMATION CONTROL SYSTEM}

\subsection{The Overall Structure}

Building automation control system is composed of the terminal hardware control part and the host computer management part. The terminal hardware control part's centre is generally the director of the building, and data is collected by various transducers and then connects with the switch of Ethernet by the programmable logic controller, and applies the star topological structure. Ethernet switch uses $100 \mathrm{M}$ bandwidths port which meets the vast requires of access to server.

The host computer management part is generally composed of prepositive server, host computer control program and workstation etc. The structure of building automation control system is shown as Fig. (1). It applies the conjunct pattern of $\mathrm{B} / \mathrm{S}$ and $\mathrm{C} / \mathrm{S}$ to monitor with $\mathrm{B} / \mathrm{S}$ in worksite and $\mathrm{C} / \mathrm{S}$ in remote control. The client and the service engineer can monitor and manage the building system anytime and anywhere. If the unit is out of order or goes wrong, the system will report the specific problem to the client and the service engineer and raise the alarm at the same time. If the system is not maintained for a long time, it will alarm the service engineer again.

With building automation control system of Ethernet computer based on the Ethernet switch and the integration with the building interface protocols such as BACnet, the site plants gather the data through the transducer and send to PLC immediately, which can contacts central control server and internet by special module. In this way, all kinds of the data of the terminal monitoring can be timely reported to the host computer of the central monitoring and the database server. Then the central monitoring will make a decision and send the solution back to the PLC. All of these accomplish the monitoring and control. This research 


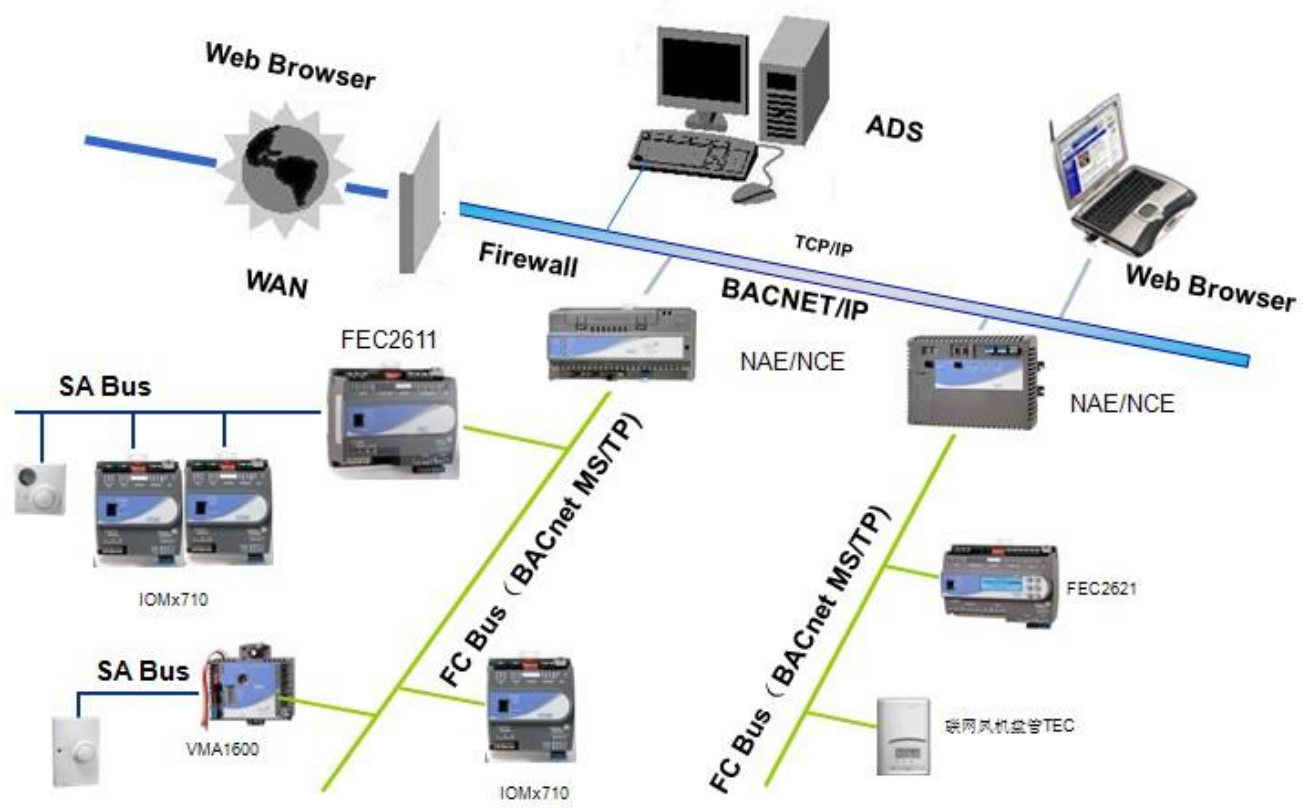

Fig. (1). The structure of building automation control system.

puts emphasis on the central air-conditioning control system whose operating principle is similar to subsystem of other control system The structure of the central air-conditioning control system can be simply divided into three levels: the first one is monitoring centre in which there is central workstation, the core of the central air-conditioning control system and generally connects with Ethernet; the second level is digital controller; the third one is the transducer which is responsible for collecting the data and is the data sources of the system.

\subsection{The Operating Principle of Ethernet}

Now Ethernet is the communication protocol standard which is most widely used in the local area network, including Ethernet, fast Ethernet and 10G Ethernet, all of which adopt CSMA/CD and comply with protocol IEEE802.3.

Generally Ethernet is star or linear structure which differs from other net by CSMA/CD that is a random communication mode. Ethernet applies the Carrier Sense Multiple Access (CSMA/CD) with collision detection. Any node in Ethernet can obtain any information in the network, so it is called broadcasting network. The working process of a node transmitting information:

(1) Monitor the information channel. If we find transmitting signal, it means that channel is busy. And we continue monitor until the signal is not busy.

(2) If we do not find the signal, we can begin to transmit the information.

(3) During the transmission, we can not stop monitor. In case of the conflict, we should back off according to the backoff algorithm. After randomly waiting a while, we can start again from step 1 .
(4) If there is no conflict, it means the success of transmission. When the other node wants to begin new information transmission, it must wait at least 9.6 microseconds after the nearest transmission. Of course these processes are running in $10 \mathrm{Mbps}$.

\subsection{The Encapsulation of Ethernet}

Actually Ethernet is also a kind of LAN, which is composed of several network nodes, connecting equipments and medium of information transmission. The communication of every node must obey standard specification above, and can be realized by the transmission medium accessing MAC. The transmission medium also conforms to corresponding standard specification. And the signal is transmitted between equipments and transmission medium. In Ethernet, the information transmission is shown by frame which is a basic unit in communication. Generally the signal cannot be allowed in the Ethernet if it is not shown in frame. And once it is found, it will be abandon. See the format of frame in physical transmission in the Ethernet in Table $\mathbf{1}$.

\section{THE DESIGN OF THE HARDWARE CONTROL SYSTEM AND THE IMPLEMENTATION OF THE SOFTWARE}

\subsection{The Design Progress of System}

Programmable logic controller or PLC is the coreequipment of building automation control system. As for the central air-conditioning control system of the PLC, we should design selectively according to specific requirements. The procedures are shown in the Fig. (2), including the sequential control of start and stop, the parameters setting and the onoff control and so on. The sequential control contains the 
Table 1. The format of frame in physical transmission in the Ethernet.

\begin{tabular}{|c|c|c|c|c|c|c|c|}
\hline PR & SA & DA & SD & TYPE & DATA & FCS & PAD \\
\hline \hline 56 place & 48 place & 48 place & 8 place & 16 place & $<=1500$ byte & 32 place & selectable \\
\hline
\end{tabular}

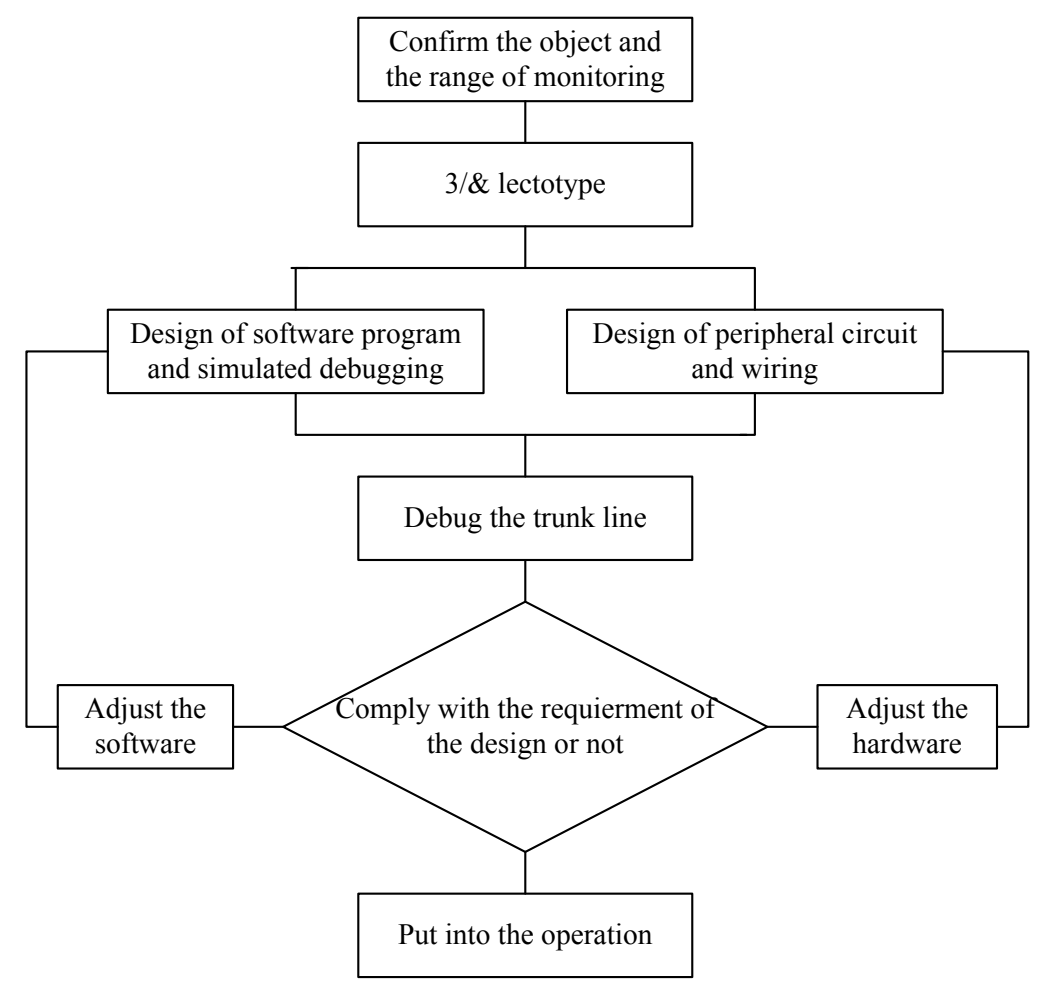

Fig. (2). The procedure of control system design.

power equipment and the auxiliary equipment. The power equipment includes the electric generator, the refrigerator and the boiler; the auxiliary equipment includes the sequential control of start and stop of the pump, the fan, and the ignition device. The parameters setting contains the inlet temperature, the outlet temperature, the high temperature and the discharge temperature of the air-conditioner and cooling water; the frequency of the solution pump and cryogen pump; the flow and the timing of air conditional water and cooling water and so on. The on-off control is mainly the operation strategy of the building system in terms of energy conservation.

\subsection{The Hardware and Input and Output Terminal}

The PLC adopted by central air conditioning control is Omron's CJ middle-sized machine whose basic plate is connected with the sequential control, the parameters setting and the on-off control and other associated equipment of building automation control system. This design adopts the CJ1WNC471 of the PLC, which is outputted by specialized relay.

In order to realize both manual and automatic operation of PLC, two buttons are added into input terminal which are a button of "start of system" and a button of "automatic/manual control". So user can start the system and choose the operational pattern by these two buttons. All of the input and output terminal of PLC is shown in the Table 2.

Meanwhile, I manage to maximize the lifetime of PLC by replacing in-out put replay with direct-current intermediate relay. In this situation, the working voltage of PLC can be $24 \mathrm{~V}$, thereby reducing the workload of PLC. And a couple of normally open contacts of direct-current intermediate relay can be treated as feedback device which is connected with signal light on the control interface.

\subsection{Data Acquisition}

This research successfully integrates the technology of the Internet of Things into system, and realizes the data acquisition monitored by transducer with the help of Ethernet. For an accurate control, we must acquire the adjustment parameters required by the system precisely, and these parameters depend on the accurate data from the transducer.

The change in system loading of the central air-condition is mainly the change of temperature, which need to be checked 
Table 2. In out put terminal of PLC.

\begin{tabular}{|c|c|c|c|}
\hline Symbol & Input Terminal & Symbol & Output Terminal \\
\hline 0 & Button of systematic switch & Y0 & Feedback of systematic switch \\
\hline 1 & Button of manual \&automatic mode & Y1 & Feedback of manual \&automatic mode \\
\hline 2 & Relay switch of absorption refrigerator & Y2 & Feedback of switch of absorption refrigerator \\
\hline 3 & Switch of gas boiler & Y3 & Feedback of switch of gas boiler \\
\hline 4 & Switch of cold water's electric valve & Y4 & Feedback of switch of cold water's electric valve \\
\hline 5 & Switch of cooling water's electric valve & Y5 & Feedback of switch of cooling water's electric valve \\
\hline 6 & Switch of boiler's hot water & Y6 & Feedback of switch of boiler's hot water \\
\hline 7 & Switch of fan in cooling water tower & Y7 & Feedback of switch of fan in cooling water tower \\
\hline 8 & Switch of pump1 of cold /hot water & Y8 & Feedback of switch of pump1 of cold /hot water \\
\hline 9 & Switch of pump2 of cold /hot water & Y9 & Feedback of switch of pump 2 of cold /hot water \\
\hline 10 & Switch of pump1 of cooling water & Y10 & Feedback of switch of pump 1 of cooling water \\
\hline 11 & Switch of pump2 of cooling water & Y11 & Feedback of switch of pump2of cooling water \\
\hline 12 & Switch of pump 1 of hot water & Y12 & Feedback of switch of pump 1 of hot water \\
\hline 13 & Switch of pump 2 of hot water & Y13 & Feedback of switch of pump 2 of hot water \\
\hline 14 & Switch of pump 1 of circling water & Y14 & Feedback of switch of pump 1 of circling water \\
\hline 15 & Switch of pump 2 of circling water & Y15 & Feedback of switch of pump 2 of circling water \\
\hline
\end{tabular}

Table 3. The simulate temperature of PLC.

\begin{tabular}{|c|c|c|c|c|}
\hline Number & Measured Parameters & Transducer & Data Acquisition Equipment & Temperature Range \\
\hline \hline 1 & Inlet temperature & PT100 & CJ safety barrier & $0 \sim 100$ \\
\hline 2 & Outlet temperature & PT100 & CJ safety barrier & $0 \sim 100$ \\
\hline 3 & Inlet temperature of hot water & PT100 & CJ safety barrier & $0 \sim 100$ \\
\hline 4 & Outlet temperature of hot water & PT100 & CJ safety barrier & $0 \sim 100$ \\
\hline 5 & Inlet temperature of cold water & PT100 & CJ safety barrier & $0 \sim 100$ \\
\hline 6 & Outlet temperature of cold water & PT100 & CJ safety barrier & $0 \sim 100$ \\
\hline 7 & Inlet temperature of second changing water & PT100 & CJ safety barrier & $0 \sim 100$ \\
\hline 8 & return temperature of air-conditioner water & PT100 & CJ safety barrier & $0 \sim 100$ \\
\hline
\end{tabular}

if we want a comprehensive monitoring and an immediate fault diagnosis. The temperature parameters are shown in Table 3.

In this system, the temperature of the water is mainly measured by the industrial platinum resistance thermometerPT100; the temperature of flue-gas is armor thermal resistance. Data Acquisition Equipment of the water temperature can apply $\mathrm{CJ}$ extended module of PLC whose range of receiving current signal is larger. A/D switch has better linear relation than the other equipment.

\subsection{Communication Equipment}

In this research, PLC of the building system is configured the RS-232 to realize the live transmission between the host computers. The port of RS232 can accomplish the point-topoint communication but fails to connect the network which 


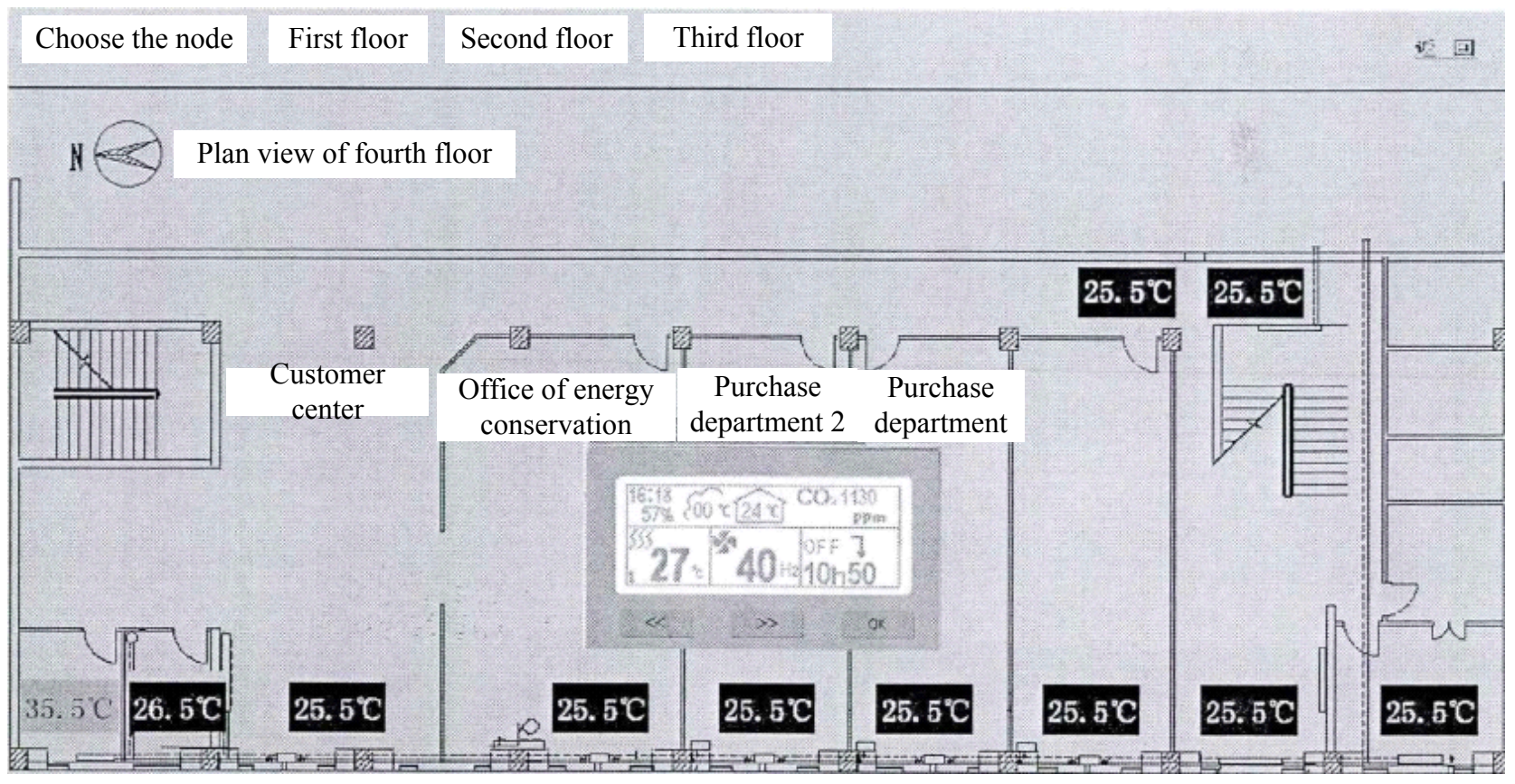

Fig. (3). The monitoring interface of the building system.

can be solved by the port of RS485. According to the practical needs, in this design each PLC controls an associated equipment of mainframe. PLC connects to RS485/RS232 switch by RS232 and then to computer room which is configured corresponding RS232/RS485 switch and the industrial personal computer by RS232. In this way, limited COM port and communication distance are solved.

\subsection{Design of PLC Master Control Module}

According to above requirements and hardware configuration, carry out the software design of PLC and mainly programme with the ladder diagram. The controlling procedure of the master control module mainly contains the setting of intercomputer communication, the transferring of the subprogram in both automatic and manual modes, and the synchronous update of each other. At the same time, modularized programming is also applied which simplifies the judge in the main program. Then through transferring each programming module, we can control the whole system. Firstly, according to the client's choice use the automatic or manual modes or both of them. As for the automatic mode, it treats time as a reference volume and reasonably control by season. So the logic of the whole procedure is clear.

\subsection{The Implementation of the Software of Host Com- puter}

The software of host computer is mainly used to immediately monitor and reasonably manage all devices of the building and its key function is data acquisition, data storage, data processing and data setting; and the function of display on screen satisfy the client's requirement of monitoring. In this study, we used the industrial personal computer of HIM; the system used VB development language; database is SQL Server. After the design the software of host computer mainly contains the following modules: general control, lighting, domestic hot water, data report and monitoring etc. The Fig. (3) is the sectional view of the building control system.

\section{THE DESIGN AND REALIZATION OF RE- MOTE NETWORK AUTOMATIC MONITORING SOFTWARE}

\subsection{The Choose of Development Platform}

Remote automatic monitored control system in this research adopts the Java platform whose advantages are: firstly, convenient operations cross the systems; secondly, scalability and reusability; thirdly, plug and play distributed calculating mode. The design of database system chooses the large-scale relational database. Because the monitoring of building has many nodes, and the quantity is big, the service platform is SQL Server2000 which facilitates the integration interface with other buildings' equipment protocols.

\subsection{Design of B/S System Interface}

According to the requirements of the monitoring, $\mathrm{B} / \mathrm{S}$ system mainly includes the following modes: the mode of air-condition mainframe, the mode of indoor unit, the mode of lighting, the mode of energy conservation, the mode of domestic hot water and the monitoring of water supply and drainage etc. Meanwhile, all of these modes have alternative choice of the background linkage boot and the building linkage boot. 


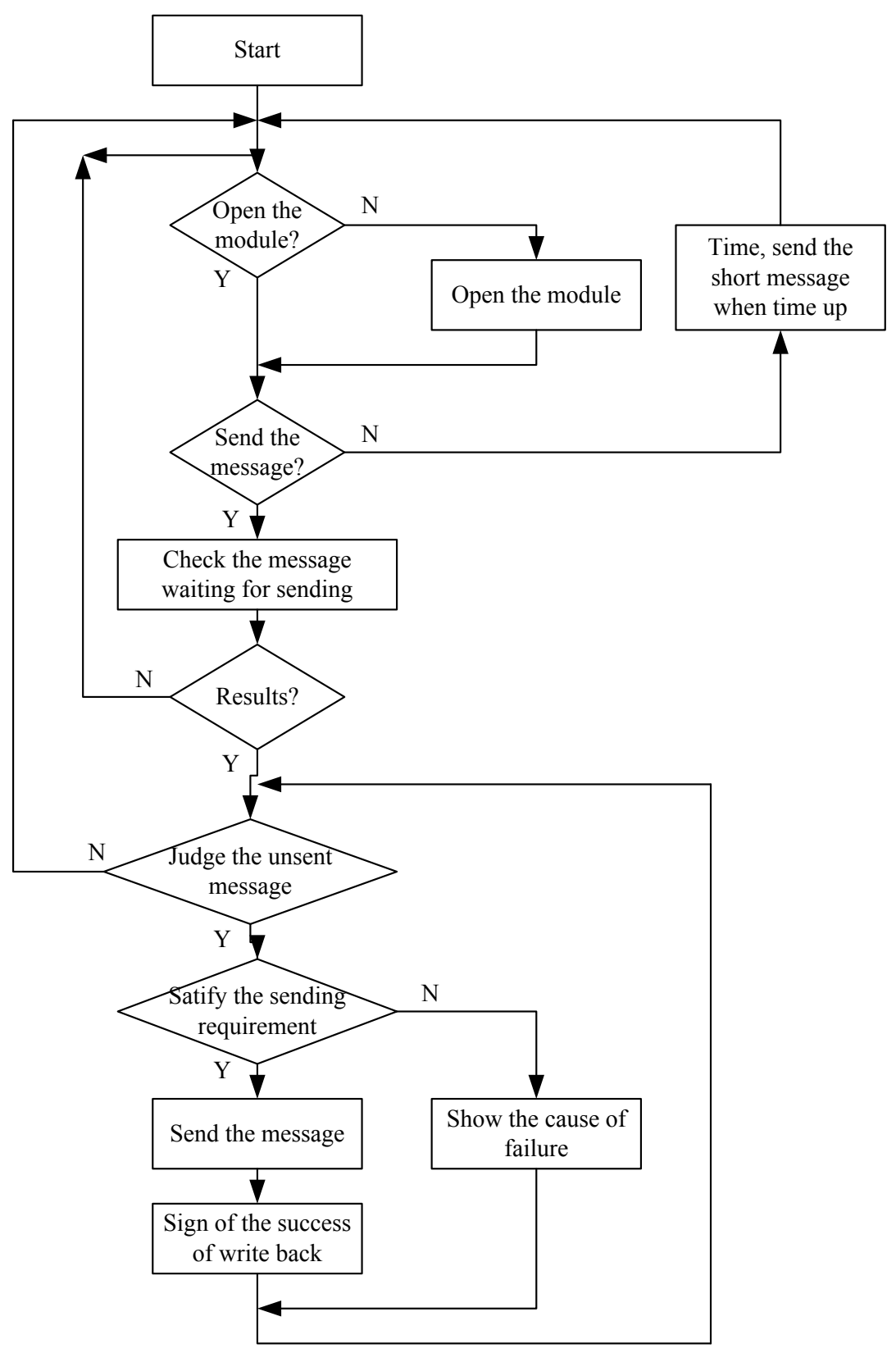

Fig. (4). The process of the short message reminder.

In this way the remote monitoring software can solely number the nodes of the building to monitor them at the same time, which increases the number of the nodes and solve the problem of limited nodes. There is the introduction of some interface:

(1) The recording interface of building monitoring nodes

After the register of building node, a set of record is set up in the monitoring center, and then we will solely number the building nodes, the building gateway and the building supervisor analysis. The remote monitoring software can track the operation.
(2) The interface of operation of building equipment

Through the code or name of node, we can immediately check the data of gateway and corresponding building equipment, such as current refrigeration mode, starting up, setting temperature, indoor temperature and power saving mode etc.

\subsection{The Auxiliary System of Short Message Alarm}

According to the requirement of the design, in case of the failure, we should use alarm to report the service engineer, 
Table 4. The parameter of short message.

\begin{tabular}{|c|c|c|}
\hline Output Parameter & Explain & Type \\
\hline \hline Uid & User identifier & String(6-12) \\
\hline Pwd & Password & String () using “, ' to separate \\
\hline Faxno & Fax number once time \\
\hline Men & Men & String (1-4) \\
\hline Title & Title & String (1-5) \\
\hline Bytes & Byte & Base64binary \\
\hline Filename & File name & String (6-10) \\
\hline Otime & Send on time & Datatime () \\
\hline
\end{tabular}

the client and the monitoring center which depends on the short message reminder. The development of short message reminder adopts the Web Service technology whose advantages are stability and cross-platform language etc. which facilitates the integration of other companies' monitoring systems with the platform of short message, and realizes the business application. The process of the short message reminder is shown in Fig. (4), and the function parameter setting of short message accessing in Table 4.

\section{CONCLUSION}

This design is focus on the automatic monitored control system of building and applies programmable logic controller (PLC) to control the spot and meanwhile realizes the remote control with the network. Because the central airconditioning control system is a typical subsystem of building automation control system, this thesis analyzes its design. The innovative points of this design:

(1) Using the Ethernet to control and solving the problem about the compatibility of instantaneity and communication protocol.

(2) The integrated design based on PLC, which realizes the data acquisition and control of the transducer and meanwhile through the technology of Internet of things successfully accomplishes the transmission and control of the terminal equipments.

(3) Remote monitoring software is added into the design which enables the system to monitor and control the equipments of the building in long-distance, and facilitate the client's control in different place, and at the same time solve the problem about the limited COM port of IPC, bad signal and less integration of network and so on.

(4) Though the design solves some problems, it is also aware of the better automatic control and there are more problems to be improved and explored: firstly, instability of the network and transmission; secondly, the integration of network; thirdly, the quantity of equipments' nodes; fourthly, operation of software system. Though these problems are solved, it is far from enough. In the future, the design of the automatic monitored control system of building can be further accomplished in terms of switching technology and 4th generation communication technology.

\section{CONFLICT OF INTEREST}

The author confirms that this article content has no conflict of interest.

\section{ACKNOWLEDGEMENTS}

Declared none.

\section{REFERENCES}

[1] Y. P. Xu and M .L. Zhou, "The Design of Intelligent Building Integrated Management system", Application Research of Computers, vol. 20, no. 6, pp. 94-96, 2003.

[2] G. G. Li, X. W. Li, and X. C. Wen, "Influence of Internet of Things Technology on the Development of Automatic Environmental Monitoring System", Environmental Monitoring in China, vol. 27 , no. 1 , pp. $5-10,2011$.

[3] J. Y. Sun and C. X. Xu, "Wireless Sensor Network Application in Oilfield Data Transmission System", Instrument Technique and Sensor, no. 6, pp. 53-57, 2012.

[4] D. Q. Song, "Application of Redundant PLC on Building Automation Control System", Industrial Control Computer, vol. 25, no. 4, pp. 45-47, 2012.

[5] H. D. He, X. R. Zhao, C. Y. Wang, and Y. B. Yang, "The Design of Serial Communication Program between PLC and Host Computer", Mechanical \& Electrical Engineering Magazine, vol. 29, no. 1, pp. 57-59, 2003.

[6] Z. L. Liu, "Fractal theory and application in city size distribution", Information Technology Journals, vol. 12, no. 17, pp. 4158-4162, 2013.

[7] B. Y. Li and Y. S. Xiao, "Present Application and Development Tendency of Networked Control System", Measurement \& Control Technology, vol. 21, no. 4, pp. 1-3, 2002. 
[8] S. Sardar and Y. H. Lee, "A Quantitative Model of Supply Chain Innovativeness as a Disruption Management Strategy in the Textile Industry", Advances in Industrial Engineering and Management, vol. 4, no. 1, pp. 9-28, 2015.

[9] J. Hu, "Ethernet and Remote monitoring network based on Internet", International Instrumentation \& Automation, vol.6, no. 2, pp. 43-45, 2002.
[10] H. Chen, F. J. Qian, and H. R. Tang, "Development and Research of the Remote Supervisory and Control System Based on Internet", Jisuanjicekongjishu, vol. 3, pp. 45-46, 2003.

[11] J. P. Li, Remote Monitoring System based on Ethernet, Journal of Zheng Zhou University (Engineering Science), vol. 23, no. 3, pp. 81-83, 2002

Received: September 16, 2014

(C) Yu et al.; Licensee Bentham Open.

This is an open access article licensed under the terms of the Creative Commons Attribution Non-Commercial License (http://creativecommons.org/licenses/by-nc/3.0/) which permits unrestricted, non-commercial use, distribution and reproduction in any medium, provided the work is properly cited. 